\title{
Offering Annual Fecal Occult Blood Tests at Annual Flu Shot Clinics Increases Colorectal Cancer Screening Rates
}

\author{
Michael B. Potter, $M D^{1}$ \\ La Phengrasamy, MPH \\ Esther S. Hudes, PbD, MPH \\ Stephen J. McPhee, $M D^{3}$ \\ Judith M.E. Walsh, MD, MPH', \\ 'Department of Family and Community \\ Medicine, University of California San \\ Francisco, San Francisco, California \\ ${ }^{2}$ Department of Epidemiology and Biostatis- \\ tics, University of California, San Francisco, \\ San Francisco, California \\ ${ }^{3}$ Department of Medicine, University of \\ California, San Francisco, San Francisco, \\ California
}

AC Annals Journal Club selection; see inside back cover or http://www. annfammed.org/AJC/.

Conflicts of interest: None of the authors bave any potential, perceived, or real conflicts of interest.

\section{CORRESPONDING AUTHOR}

Michael B. Potter, MD

Department of Family

and Community Medicine

Box 0900, UCSF

San Francisco, CA 94143

potterm@fcm.ucsf.edu

\begin{abstract}
PURPOSE We wanted to determine whether providing home fecal occult blood test (FOBT) kits to eligible patients during influenza inoculation (flu shot) clinics can contribute to higher colorectal cancer screening (CRCS) rates.

METHODS The study was time randomized. On 8 dates of an annual flu shot clinic at the San Francisco General Hospital, patients were offered flu shots as usual (control group) and on 9 other dates, patients were offered both flu shots and FOBT kits (intervention group).

RESULTS The study included 514 patients aged 50 to 79 years, with 246 in the control group and 268 in the intervention group. At the conclusion of flu season, FOBT screening rates increased by 4.4 percentage points from $52.9 \%$ at baseline to $57.3 \%(P=.07)$ in the control group, and increased by 29.8 percentage points from $54.5 \%$ to $84.3 \%(P<.001)$ in the intervention group, with the change among intervention participants 25.4 percentage points greater than among control participants ( $P$ value for change difference $<.001)$. Among patients initially due for CRCS, $20.7 \%$ in the control group and $68.0 \%$ in the intervention group were up-to-date at the conclusion of the study $(P<.001)$. In multivariate analyses, the odds ratio for becoming up-to-date with screening in the intervention group (vs the control group) was 11.3 (95\% Cl, 5.8-22.0).
\end{abstract}

CONCLUSIONS Offering FOBT kits during flu shot clinics dramatically increased the CRCS rate for flu shot clinic attendees. Pairing home FOBT kits with annual flu shots may be a useful strategy to improve CRCS rates in other primary care or public health settings.

Ann Fam Med 2009;7:17-23. DOI: 10.1370/afm.934

\section{INTRODUCTION}

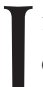
ncidence of and mortality from colorectal cancer can be reduced with colorectal cancer screening (CRCS). ${ }^{1}$ In the United States, recommended CRCS tests have long included the annual, guaiac-based, home fecal occult blood test (FOBT), flexible sigmoidoscopy every 5 years, annual FOBT plus flexible sigmoidoscopy every 5 years, doublecontrast barium enema every 5 years, or colonoscopy every 10 years. ${ }^{2,3}$ Recently, fecal immunochemical testing, stool DNA testing, and computed tomographic colonoscopy have also been endorsed as screening methods. ${ }^{4}$ Despite these testing options, only slightly more than one-half of eligible adults aged 50 years and older report being up-to-date with CRCS. ${ }^{5}$ Partly as a result, colorectal cancer remains the second leading cause of cancer death in the United States. ${ }^{6}$ Primary care remains the most important access point for $\mathrm{CRCS}^{7}{ }^{7}$ but primary care offices frequently lack systems to ensure that CRCS is offered when needed and to support patient adherence to tests that are ordered ${ }^{8-12}$

Providing CRCS where uninsured or underinsured patients receive 
care is accompanied by special challenges. Financial constraints often limit the menu of screening options to FOBT, the least expensive CRCS test, with colonoscopy reserved for cases of abnormal FOBT results or other high-risk conditions. ${ }^{13}$ The barriers to providing an annual FOBT may be magnified by educational, linguistic, ethnic, social, and cultural factors. ${ }^{14-18}$ Intensive educational interventions conducted in person or by telephone can increase FOBT rates, but they may be impractical for resource-limited settings. ${ }^{19-21}$ Lessintensive interventions, such as mailing FOBT kits to patients, have been tried, with completion rates ranging from $1 \%$ for an intervention involving no patient follow-up to about $40 \%$ for a system of mailing FOBT kits to patients 2 weeks before a scheduled appointment. ${ }^{21-23}$

Successful and sustainable primary care approaches to achieve high FOBT completion rates may require multiple strategies, including simple office systems that enable nonphysician clinic staff to identify and personally offer FOBTs to eligible patients at least once a year. ${ }^{24,25}$ One strategy, recently proposed, includes offering FOBTs in conjunction with annual influenza inoculations (flu shots). ${ }^{26}$ This clinical trial is the first to test this approach by using clinic staff to provide brief CRCS education and FOBT kits to eligible patients during annual primary care-based flu shot clinics.

\section{METHODS}

\section{Theoretical Framework}

The General Model of the Determinants of Behavioral Change, a synthesis of behavioral theories, asserts that the performance of a desired health behavior is most influenced by a person's attitudes toward the behavior in question; perceived community norms that relate to the behavior; the person's self-efficacy, skills, and abilities related to performing the behavior; and other environmental conditions that influence the person's intention or ability to perform the desired behavior. ${ }^{27}$ The intervention was designed to influence attitudes, expectations, and knowledge about CRCS in a diverse patient population by offering FOBT with brief educational counseling and reminder calls in association with another clinically accepted annual preventive health service that is already desired by the patient.

\section{Study Setting}

The Family Health Center is a residency-based clinic at San Francisco General Hospital staffed by 41 family medicine residents, 26 faculty physicians, and 6 nurse-practitioners who conduct more 38,000 patient visits per year. The clinic population is economically disadvantaged and ethnically diverse, including mostly Asian, Latino, and Eastern European immigrants, as well as US-born African Americans and whites, nearly all of whom live near or below the federal poverty level. Each autumn, the Family Health Center provides flu shot clinics run by multilingual medical assistants and health workers.

\section{Study Population and Randomization}

In September 2006, patients aged 50 to 79 years were mailed multilingual flu shot campaign announcements in English, Chinese, Russian, Spanish, and Vietnamese. Patients were told they could get a flu shot at their next regular clinic visit or during 1 of 17 half-day flu shot clinic sessions between October and December. To reduce contamination between intervention and control group patients, the study enrolled only patients attending the $17 \mathrm{flu}$ shot clinic sessions. We randomly selected 8 of the 17 flu shot clinic sessions in blocks of 2 and 3 for provision of flu shots only (control group), and 9 sessions for flu shots plus provision of FOBT kits for patients who would be eligible for CRCS by the end of flu shot season in March 2007.

Eligible patients had not had a FOBT since the end of the prior influenza season, a colonoscopy in the past 10 years, any previously unevaluated abnormal FOBT results, or a history of recent unevaluated rectal bleeding. Some study patients may have been at higher than average risk and therefore eligible for colonoscopy instead of FOBT, but it was decided to leave the responsibility for risk assessment to primary care clinicians and allow unscreened patients with unknown family history to participate in this study. Patients who had only flexible sigmoidoscopy in the past 5 years were also considered eligible for FOBT, because many guidelines endorse FOBT and flexible sigmoidoscopy together. ${ }^{2-4}$

The primary study outcome was change in CRCS up-to-date status from being due for a screening test, defined as not having a FOBT in the last year, a flexible sigmoidoscopy or double-contrast barium enema in the past 5 years, a colonoscopy in the past 10 years, or having any previously unevaluated abnormal FOBT results or recent unevaluated rectal bleeding, to having completed the FOBT. Patients were not told in advance that FOBT might be offered along with their flu shots. The clinic staff was not told in advance on what dates they would perform the intervention. All participants were established primary care patients, so intervention effectiveness was measured as an addition to usual care. Figure 1 displays a flow diagram of study participants.

\section{Staff Training}

The researchers (M.P. and L.P.) conducted 2, 1-hour staff training sessions, 1 of which included role playing. After role playing, the staff identified best practices for discussing CRCS with patients from different cultural 


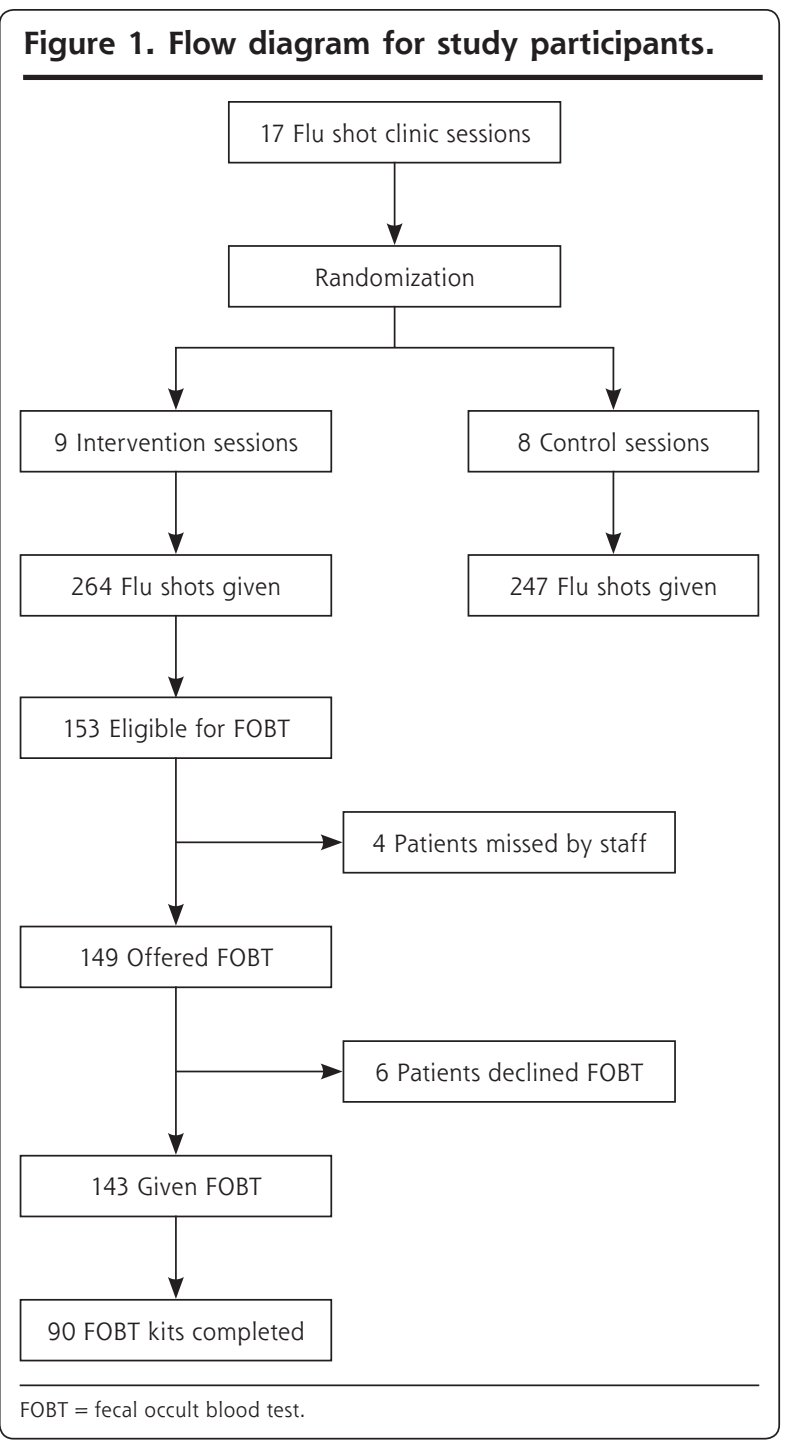

backgrounds and suggested improvements in patient education materials. Another 1-hour staff meeting was conducted 2 weeks into the intervention to answer questions and assure research protocol fidelity.

\section{Intervention Procedures}

The day before each flu shot clinic, a member of the research team (L.P.) reviewed the electronic medical records of scheduled patients to determine who was eligible for FOBT. The list of eligible patients was provided to the flu shot clinic staff so they would know in advance who needed FOBT. On arrival in the clinic, patients received a colorful multilingual education sheet with the message: "Flu is Preventable! Colon Cancer is Preventable! Yearly Colon Testing Saves Lives! All our doctors and nurses recommend yearly Colon Testing for adults aged 50 to 79 . When should you get tested? We will tell you today!" Names of all clinicians and staff were listed on the back of the multilingual education sheet. After their flu shots, eligible patients were offered an FOBT kit (Hemoccult II, Beckman Coulter, Fullerton, California) with 6th- grade reading level instructions as defined in English with the Fry Readability Index. ${ }^{28,29}$ Instructions were provided in English on 1 side and 1 of 4 other languages (Chinese, Russian, Spanish, or Vietnamese) on the reverse. FOBT kits were provided with postagepaid return envelopes. Patients were telephoned if they had not returned their completed kit at 3 weeks and again at 6 weeks after the FOBT kit was dispensed.

\section{Data Analysis}

We created a database that included participant birth date, sex, ethnicity, primary language, insurance status, and annual income obtained from administrative databases, as well as dates of hospital and clinic visits and dates of most recent FOBT, flexible sigmoidoscopy, double-contrast barium enema, and colonoscopy obtained from the electronic medical record. Records of control and intervention patients who were identified as having a previously unevaluated abnormal FOBT result were flagged so the patients would not be categorized as being due for repeat screening FOBT in any of our analyses (also so they could be referred directly for colonoscopy by their primary care clinicians).

Data analyses were conducted using Stata, version 9 (Statacorp LP, College Station, Texas). We compared baseline characteristics of the intervention and control groups using the 2-sample $t$ test for continuous variables and the Pearson $\chi^{2}$ test for categorical variables. To compare the changes in CRCS status between the 2 study arms, a $-1,0,+1$ score was created for the preintervention to postintervention change for each patient. In this scoring system, +1 indicates being due for screening at preintervention and up-to-date at postintervention ${ }_{i}-1$ indicates being up-to-date at preintervention and due for screening at postintervention, and 0 indicates no preintervention to postintervention change in CRCS status.

A 2-sample Wilcoxon test was used to compare these change scores for the 2 groups. Within each arm, the McNemar test was used to compare preintervention to postintervention percentage point change in CRCS status. At each time point, group differences in CRCS status were assessed using the Pearson $\chi^{2}$ test. We used the Pearson $\chi^{2}$ test to compare the 2 arms within 2 strata: (1) patients not up-to-date for CRCS at the beginning of the intervention, and (2) patients up-to-date with CRCS at the beginning of the intervention. Using available preselected predictor variables that have been associated with variations in screening completion, ${ }^{30-34}$ we next explored predictors of CRCS 
with multivariate logistic regression models. Retained covariates were age, sex, ethnicity, primary language, insurance status, income, and number of primary care visits in the previous year. The University of California, San Francisco, Committee on Human Research approved the study.

\section{RESULTS}

\section{Demographics}

Participant characteristics are displayed in Table 1. The study participants included more women than men and was ethnically diverse. Fifteen different primary languages were represented. Besides English, most primary languages were Spanish (24\%), Vietnamese (20\%), Cantonese (17\%), and Russian (4\%). Most patients were insured through Medicare or Medicaid, but about onefifth were uninsured. Most had more than 5 clinic visits

in the last year, but only about one-half had CRCS within recommended time intervals. The intervention and control groups were similar, except the intervention group was younger (mean age 63.7 vs 65.6 years, $P=.004)$ and had lower self-reported annual income (mean income $\$ 9,785$ vs $\$ 10,967, P=.036$ ).

\section{FOBT Kit Distribution}

In the intervention group, 153 of the 268 patients were eligible for FOBT (Figure 1). Among these patients, 122 were eligible and overdue for FOBT because they had no CRCS noted in the electronic medical record within recommended intervals and no unevaluated abnormal FOBT result 31 more were considered eligible but not necessarily overdue for FOBT because either they had had flexible sigmoidoscopy in the past 5 years but no FOBT in the last year or because they would become due for CRCS by the end of the flu season without a new test. No patients

Table 1. Demographic Characteristics of Study Participants $(\mathrm{N}=514)$ in the Control and Intervention Groups

\begin{tabular}{|c|c|c|c|}
\hline Characteristics & $\begin{array}{c}\text { Control } \\
(n=246)\end{array}$ & $\begin{array}{l}\text { Intervention } \\
(n=268)\end{array}$ & $\begin{array}{c}P \\
\text { Value }\end{array}$ \\
\hline Age, mean (SD), years & $65.6(7.4)$ & $63.7(7.6)$ & $.004^{\mathrm{a}}$ \\
\hline Female sex, \% & 65.9 & 61.2 & $.273^{b}$ \\
\hline Ethnicity, \% & & & $.142^{\mathrm{b}}$ \\
\hline African American & 6.1 & 5.6 & \\
\hline Asian/Pacific Islander & 56.1 & 48.1 & \\
\hline Latino & 25.2 & 35.8 & \\
\hline Non-Latino white & 9.8 & 7.8 & \\
\hline Other/unknown & 2.8 & 2.6 & \\
\hline Non-English primary language, $\%$ & 75.6 & 72.0 & $.392^{\mathrm{b}}$ \\
\hline Economic indicator: insurance, \% & & & $.638^{b}$ \\
\hline Medicare & 43.5 & 40.3 & \\
\hline Medicaid & 37.0 & 38.4 & \\
\hline Uninsured & 17.9 & 20.5 & \\
\hline Other & 1.6 & 0.8 & \\
\hline $\begin{array}{l}\text { Economic indicator: yearly income, } \\
\text { mean (SD), \$ }\end{array}$ & $\begin{array}{l}10,967 \\
(6,377)\end{array}$ & $\begin{array}{c}9,785 \\
(6,383)\end{array}$ & $.036^{\mathrm{a}}$ \\
\hline \multicolumn{4}{|l|}{ Health care access in the last year } \\
\hline Primary care visits, mean (SD), No. & $6.0(3.7)$ & $5.4(3.5)$ & $.091^{\mathrm{a}}$ \\
\hline One or more hospitalizations, \% & 4.9 & 8.2 & $.129^{b}$ \\
\hline $\begin{array}{l}\text { One or more emergency department } \\
\text { visits, \% }\end{array}$ & 7.3 & 10.4 & $.214^{b}$ \\
\hline \multicolumn{4}{|l|}{$\begin{array}{l}\text { Preintervention flu shot and CRCS } \\
\text { status, \% }\end{array}$} \\
\hline Received flu shot in last year & 68.7 & 69.8 & $.792^{b}$ \\
\hline \multicolumn{4}{|l|}{ Baseline status for CRCS tests, \% } \\
\hline FOBT in last 12 months & 36.2 & 35.4 & $.863^{b}$ \\
\hline FS in last 5 years & 9.8 & 10.5 & $.795^{b}$ \\
\hline DCBE in last 5 years & 1.6 & 1.1 & $.621^{b}$ \\
\hline Colonoscopy in last 10 years & 16.3 & 17.2 & $.784^{b}$ \\
\hline \multicolumn{4}{|c|}{$\begin{array}{l}\text { CRCS = colorectal cancer screening; } D C B E=\text { double-contrast barium enema; } F O B T=\text { fecal occult } \\
\text { blood test; FS = flexible sigmoidoscopy. }\end{array}$} \\
\hline $\begin{array}{l}\text { a } 2 \text {-sample } t \text { test. } \\
\text { b Pearson } \gamma^{2} \text { test }\end{array}$ & & & \\
\hline
\end{tabular}
reported rectal bleeding. In the intervention group, 14 patients were categorized as ineligible for FOBT because they had a previously unevaluated FOBT result compared with 19 patients in the control group. The remaining 101 patients were ineligible for FOBT because they had had a colonoscopy in the past 10 years $(46$ patients), normal findings on a double-contrast barium enema in the past 5 years $(2$ patients), or a normal findings on a home FOBT since the conclusion of the last year's flu season (53 patients). The staff provided FOBT kits to 143 of the 153 eligible patients.

\section{Preintervention and Postintervention Screening Rates}

Table 2 displays preintervention and postintervention changes in the percentage of patients up-to-date with CRCS in the control and intervention groups. The CRCS rate increased more in the intervention than in the control group. Table 3 shows that most of the intervention effect resulted from increased screening among intervention patients who were initially due for screening. Because of limited access to endoscopy and double-contrast barium enema in this setting, this increase was the result of increased completion of FOBT. Intervention patients who were initially up-to-date were also more likely to remain so at the end of the study compared with similar control patients. 


\begin{tabular}{|c|c|c|c|}
\hline CRCS Status & $\begin{array}{l}\text { Control } \\
(n=246)\end{array}$ & $\begin{array}{l}\text { Intervention } \\
(n=268)\end{array}$ & $\begin{array}{l}\text { Between } \\
\text { Group } \\
P \text { Value }\end{array}$ \\
\hline $\begin{array}{l}\text { CRCS up-to-date before influenza } \\
\text { season (October 16, 2006), \% }\end{array}$ & 52.9 & 54.5 & $.711^{\mathrm{a}}$ \\
\hline $\begin{array}{l}\text { CRCS up-to-date after influenza } \\
\text { season (March 31, 2007), \% }\end{array}$ & 57.3 & 84.3 & $<.001^{\mathrm{a}}$ \\
\hline Percentage point change & $\begin{array}{l}+4.4(-0.7 \\
\quad \text { to } 9.7)\end{array}$ & $\begin{array}{l}+29.8(23.7 \\
\quad \text { to } 36.0)\end{array}$ & $<.001^{\mathrm{b}}$ \\
\hline $\begin{array}{l}\text { Preintervention to postinterven- } \\
\text { tion } P \text { value }\end{array}$ & .071 & $<.001$ & \\
\hline \multicolumn{4}{|l|}{ CRCS = colorectal cancer screening. } \\
\hline \multicolumn{4}{|c|}{$\begin{array}{l}\text { a Pearson } \chi^{2} \text { test. } \\
\text { b } 2 \text {-sample Wilcoxon rank-sum test on the preintervention-postintervention difference scores. } \\
\text { 'McNemar test. }\end{array}$} \\
\hline
\end{tabular}

diagnostic colonoscopy include lack of accurate contact information for the patient, delays in colonoscopy scheduling, and patient refusal or failure to complete scheduled colonoscopy appointments.

\section{DISCUSSION}

This study shows that medical assistants and health workers can be successful in offering FOBT kits to eligible patients during a primary care-based annual flu shot campaign. CRCS rates increased dramatically among patients exposed to the intervention. In the control group, there was a nonsignificant increase in CRCS, possibly because of temporal trends toward increased screening, contamination related

\section{Multivariate Logistic Regression for Subgroups} Initially Due for or Up-to-Date With CRCS

Multivariate analyses are displayed in Table 4 . These analyses confirmed that intervention patients were more likely than control patients to be up-to-date with CRCS when the study ended, regardless of baseline CRCS status. Patients initially due for screening who had higher self-reported income and more primary care visits in the previous year were also more likely to be up-to-date with screening at the end of the study.

\section{FOBT Findings and Follow-Up}

Of 90 intervention patients who returned kits as part of the intervention, $4(5 \%)$ had positive test results and were referred for colonoscopy, as were the 33 patients who were noted to have had a previously unevaluated FOBT. Colonoscopy was obtained for all 4 intervention patients whose results were positive but for only 16 of the remaining 33 patients with a previously unevaluated positive FOBT result. Of the 20 patients who completed a colonoscopy, 9 had tubular adenomas removed, with no cancers detected. Efforts to obtain colonoscopy for the remaining 17 patients with unevaluated positive FOBT results continue. Reasons for inability to obtain

\begin{tabular}{|c|c|c|c|}
\hline Study Participants & $\begin{array}{l}\text { Control } \\
\text { No. (\%) }\end{array}$ & $\begin{array}{c}\text { Intervention } \\
\text { No. (\%) }\end{array}$ & $P$ Value $^{\mathrm{a}}$ \\
\hline Total patients initially not up-to-date & 116 & 122 & \\
\hline Patients who became up-to-date & $24(20.7)$ & $83(68.0)$ & $<.001$ \\
\hline Total patients initially up-to-date & 130 & 146 & \\
\hline Patients who remained up-to-date & $117(90.0)$ & $143(98.0)$ & $<.005$ \\
\hline
\end{tabular}

to intervention and control participants attending the same clinic site, or chance.

The intervention group was somewhat younger and poorer than the control group. In multivariate analyses accounting for these differences, however, the intervention remained a strong predictor of CRCS. Higher income and more primary care visits, but not age, were other predictors of becoming up-to-date with CRCS for this cohort. The association between higher income and becoming up-to-date with CRCS must be interpreted cautiously, because nearly all study participants reported very low income.

When considering the potential effectiveness of our intervention in other clinical settings, several issues deserve consideration. First, despite the simplicnot without complexity. Key elements included use of electronic medical records to identify which patients were due for CRCS; trained multilingual staff to carry out the intervention; multilingual low-literacy patient education materials explaining the purpose of CRCS and how to complete the FOBT; free FOBT kits and postage-paid return envelopes; and reminder calls at 3 and 6 weeks, when needed. Before the start of each flu shot clinic, the research assistant took about 1 minute per patient to determine CRCS status using the electronic medical record, and health care workers took about 2 minutes per patient to offer FOBT kits to elegible patients. A level of staff commitment similar to that needed to execute the flu shot clinic itself may be required to implement the CRCS intervention successfully. Nonetheless, in settings where FOBTs and flu ity of the concept, execution of the intervention is 


\begin{tabular}{|c|c|c|}
\hline Predictor Variable & $\begin{array}{l}\text { Patients Initially } \\
\text { Overdue for } \\
\text { CRCS }(n=238) \\
\text { OR }(95 \% \mathrm{Cl})\end{array}$ & $\begin{array}{l}\text { Patients Initially } \\
\text { Up-to-Date for } \\
\text { CRCS }(n=276) \\
\text { OR }(95 \% \mathrm{Cl})\end{array}$ \\
\hline Study arm, intervention (vs control) & $11.3(5.8-22.0)^{\mathrm{a}}$ & $5.8(1.5-22.0)^{\mathrm{a}}$ \\
\hline Age, $50-64$ y (vs $65-79$ y) & $0.8(0.4-1.5)$ & $1.0(0.3-3.4)$ \\
\hline Sex, male (vs female) & $1.1(0.6-2.1)$ & $2.5(0.7-9.3)$ \\
\hline Ethnicity, Hispanic (vs Asian) & $0.8(0.4-1.6)$ & $0.4(0.1-1.3)$ \\
\hline Other (vs Asian) & $0.5(0.2-1.1)$ & $1.7(0.2-15.9)$ \\
\hline Primary language, English (vs non-English) & $0.8(0.4-1.8)$ & $2.0(0.4-10.0)$ \\
\hline Insurance, insured (vs uninsured) & $1.4(0.6-3.2)$ & $1.3(0.3-5.2)$ \\
\hline Income, above median (vs below) & $2.0(1.1-3.8)^{\mathrm{b}}$ & $0.7(0.2-2.0)$ \\
\hline $\begin{array}{l}\text { Primary care visits, above median } \\
\text { (vs below median) }\end{array}$ & $2.0(1.0-3.7)^{b}$ & $0.7(0.2-2.3)$ \\
\hline \multicolumn{3}{|l|}{ CRCS = colorectal cancer screening; $\mathrm{OR}=$ odds ratio. } \\
\hline \multicolumn{3}{|l|}{$\begin{array}{l}\text { a } P<.001 \text { for comparison with reference category. } \\
\text { b } P<.05 \text { for comparison with reference category. }\end{array}$} \\
\hline
\end{tabular}

tion. We discovered, however, there were other patients with previously unevaluated abnormal FOBT results, in some cases more than 3 or 4 years in the past. Despite the commitment of clinic staff, only about one-half of those patients have thus far been provided with diagnostic colonoscopy. FOBT-based CRCS programs such as ours will not be capable of reaching their full potential to improve health outcomes without prompt access to diagnostic colonoscopy when needed.

National efforts to provide flu shots to all adults older than 50 years should ultimately increase the proportion of CRCS-eligible adults who could benefit from a combined flu shot-FOBT program. ${ }^{35}$ Advertising the availability and importance of an annual FOBT along with flu shots

shots are of high priority, combining efforts to support both activities simultaneously may be time-efficient and worthwhile.

A second issue is the potential reach of this intervention. Only some patients who need CRCS receive annual flu shots during dedicated flu shot clinics. For example, at the San Francisco General Hospital Family Health Center, only about one-half of patients who are due for CRCS typically get annual flu shots at the clinic, and only about one-half of those getting flu shots at the clinic get them during dedicated flu shot sessions; the remainder get them during other visits. We estimate that, if our intervention were adapted and expanded to reach all flu shot recipients with similar acceptance rate, the total CRCS rate for the entire eligible clinic population could improve by up to 15 percentage points over baseline. If the number of flu shot recipients increased, the potential to increase CRCS rates with our combined flu shot-FOBT program would also increase.

Third, patients who attend flu shot clinics could be more interested in preventive care and willing to accept FOBT than other clinic patients. Even so, the baseline CRCS rate for all 2,768 Family Health Center primary care patients between the ages of 50 and 79 years was $48.6 \%$, only slightly lower than baseline CRCS rates for the subset of clinic patients who were the target of our study, which suggests that patients attending flu shot clinic are not more inclined to participate in CRCS than other clinic patients.

A final issue critical for the success of any FOBT program is follow-up of abnormal results. We provided diagnostic colonoscopy to the 4 patients whose test results were positive as part of the study interven- could become an efficient way to increase patient interest in both procedures. Establishing flexible and timeefficient protocols for nonphysician staff to manage this program independently could increase health care provider interest in its adoption, implementation, and maintenance. For example, a few brief questions can establish patient eligibility for FOBT with reasonable accuracy in diverse populations. ${ }^{36-38}$ Electronic medical records with preventive health prompts that are accessible to nonphysician staff are becoming more common, even in safetynet settings. ${ }^{39}$ We are studying these and additional adaptations to extend the reach and effectiveness of the flu shot-FOBT program at the San Francisco Department of Public Health and in other clinical settings.

In conclusion, annual flu shot activities represent an opportunity for nonphysician staff to offer a FOBT to eligible patients who need it at a time when they are already thinking about annual prevention. Combining annual FOBT and flu shot activities could become a powerful way to promote CRCS in primary care settings, especially in communities and clinics where FOBT remains the primary screening option.

To read or post commentaries in response to this article, see it online at http://www.annfammed.org/cgi/content/full/7/1/17. mary health care; delivery of health care; health care disparities; minority health

Submitted February 16, 2008; submitted, revised, August 7, 2008; accepted August 13, 2008.

Findings from this study have been presented at the North American Primary Care Research Group, October 2007, and the Bay Area Clinical Research Symposium, November 2007.
Key words: Colorectal cancer; mass screening; health promotion; pri- 
Funding support: This study was funded by grants from the American Cancer Society, Cancer Control Career Development Award for Primary Care Physicians, and the National Cancer Institute, Asian American Network for Cancer Awareness, Research \& Training.

Acknowledgments: The authors would like to acknowledge the entire staff at the San Francisco General Hospital's Family Health Center for supporting the implementation of this project within the context of a busy clinical setting.

\section{References}

1. Walsh JM, Terdiman JP. Colorectal cancer screening: scientific review. JAMA. 2003;289(10):1288-1296.

2. U.S. Preventive Services Task Force. Screening for colorectal cancer: recommendation and rationale. Ann Intern Med. 2002;137(2):129-131.

3. Smith RA, Cokkinides V, Eyre HJ. Cancer screening in the United States, 2007: a review of current guidelines, practices, and prospects. CA Cancer J Clin. 2007;57(2):90-104.

4. Levin B, Lieberman DA, McFarland B, et al.; for the American Cancer Society Colorectal Cancer Advisory Group, the US Multi-Society Task Force, and the American College of Radiology Colon Cancer Committee. Screening and surveillance for the early detection of colorectal cancer and adenomatous polyps, 2008: A joint guideline from the American Cancer Society, the US Multi-Society Task Force on Colorectal Cancer, and the American College of Radiology. CA Cancer J Clin. 2008;58(3):130-160.

5. Centers for Disease Control. Increased use of colorectal cancer tests - United States, 2002 and 2004. MMWR Morb Mortal W/kly Rep. 2006;55(11):308-311.

6. Jemal A, Siegel R, Ward E, Murray T. XuJ, Thun MJ. Cancer statistics, 2007. CA Cancer J Clin. 2007;57(1):43-66.

7. Wender RC. Preserving primary care: the front line in the war against cancer. CA Cancer J Clin. 2007;57(1):4-5.

8. Yarnall KS, Pollack KI, Ostbye T, Krause KM, Michener L. Primary care: is there enough time for prevention? Am J Public Health. 2003;93(4):635-641.

9. Ostbye T, Yarnall KS, Krause KM, Pollak KI, Gradison M, Michener $\mathrm{JL}$. Is there time for management of patients with chronic diseases in primary care? Ann Fam Med. 2005;3(3):209-214.

10. Walsh JM, McPhee SJ. A systems model of clinical preventive care: an analysis of factors influencing patient and physician. Health Educ Q. 1992;19(2):157-175.

11. Guerra CE, Schwartz JS, Armstrong K, Brown JS, Halbert CH, Shea JA. Barriers of and facilitators to physician recommendation of colorectal cancer screening. J Gen Intern Med. 2007;22(12):1681-1688.

12. Beydoun HA, Beydoun MA. Predictors of colorectal cancer screening behaviors among average-risk older adults in the United States. Cancer Causes Control. 2008;19(4):339-359.

13. Fisher JA, Fikry C, Troxel AB. Cutting cost and increasing access to colorectal cancer screening: another approach to following guidelines. Cancer Epidemiol Biomarkers Prev. 2006;15(1):108-113.

14. O'Malley AS, Beaton E, Yabroff KR, Abramson R, Mandelblatt J. Patient and provider barriers to colorectal cancer screening in the primary care safety-net. Prev Med. 2004;39(1):56-63.

15. Walsh JM, Kaplan CP, Nguyen B, Glidengorin G, McPhee SJ, PerezStable EJ. Barriers to colorectal cancer screening in Latino and Vietnamese Americans compared with non-Latino white Americans. J Gen Intern Med. 2004;19(2):156-166.

16. Wang JH, Liang W, Chen MY, et al. The influence of culture and cancer worry on colon cancer screening among older ChineseAmerican women. Ethn Dis. 2006;16(2):404-411.

17. Goel MS, Wee CC, McCarthy EP, Davis RB, Ngo-Metzger Q, Phillips RS. Racial and ethnic disparities in cancer screening: the importance of foreign birth as a barrier to care. J Gen Intern Med. 2003;18(12):1028-1035.
18. Tu SP, Talyor V, Yasui Y, et al. Promoting culturally appropriate colorectal cancer screening through a health educator: a randomized controlled trial. Cancer. 2006;107(5):959-966.

19. Stokamer CL, Tenner CT, Chaudhuri J, Vazquez E, Bini EJ. Randomized controlled trial of the impact of intensive patient education on compliance with fecal occult blood testing. J Gen Intern Med. 2005;20(3):278-282.

20. Beach ML, Flood AB, Robinson CM, et al. Can language-concordant prevention care managers improve cancer screening rates? Cancer Epidemiol Biomarkers Prev. 2007;16(10):2058-2064.

21. Walsh JM, Salazar R, Terdiman JP, Gildengorin G, Perez-Stable EJ. Promoting use of colorectal cancer screening tests. Can we change physician behavior? J Gen Intern Med. 2005;20(12):1097-1101.

22. Church TR, Yeazel MW, Jones RM, et al. A randomized trial of direct mailing of fecal occult blood tests to increase colorectal cancer screening. J Natl Cancer Inst. 2004;96(10):770-778.

23. Goldberg D, Schiff GD, McNutt R, Furumoto-Dawson A, Hammerman M, Hoffman A. Mailings timed to patients' appointments: a controlled trial of fecal occult blood test cards. Am J Prev Med. 2004;26(5):431-435.

24. Sarfaty M, Wender R. How to increase colorectal cancer screening rates in practice. CA Cancer J Clin. 2007;57(6):354-366.

25. Klabunde CN, Lanier D, Breslau ES, et al. Improving colorectal cancer screening in primary care practice: innovative strategies and future directions. J Gen Intern Med. 2007;22(8):1195-1205.

26. Klabunde CN, Meissner HI, Wooten KG, Breen N, Singleton JA. Comparing colorectal cancer screening and immunization status in older Americans. Am J Prev Med. 2007;33(1):1-8.

27. Institute of Medicine. Committee on Communication and Behavior Change in the 21st Century: Improving the health of Diverse Populations, Board on Neuroscience and Behavioral Health. Speaking of Health: Assessing Health Communication Strategies For Diverse Populations. Washington, DC: National Academies Press; 2002.

28. Friedman DB, Hoffman-Goetz L. A systematic review of readability and comprehension instruments used for print and web-based cancer information. Health Educ Behav. 2006;33(3):352-373.

29. Fry E. A readability formula that saves time. J Read. 1968;11(7):513-516.

30. Zimmerman RK, Norwalk MP, Tabbarah M, Grufferman S. Predictors of colorectal cancer screening in diverse primary care practices. BMC Health Serv Res. 2006;6:116-124.

31. Wong ST, Gildengorin G, Nguyen T, Mock J. Disparities in colorectal cancer screening rates among Asian Americans and non-Latino whites. Cancer. 2005;104(12)(Suppl):2940-2947.

32. Iaonnou GN, Chapko MK, Dominitz JA. Predictors of colorectal cancer screening participation in the United States. Am J Gastroenterol. 2003;98(9):2082-2091.

33. Zapka JG, Puleo E, Vickers-Lahti M, Luckmann R. Healthcare system factors and colorectal cancer screening. Am J Prev Med. 2002;23(1):28-35.

34. Shih YC, Elting LS, Levin B. Disparities in colorectal screening between US-born and foreign-born populations: evidence from the 2000 National Interview Survey. J Cancer Educ. 2008;23(1):18-25.

35. US Department of Health and Human Services. Healthy People 2010. Washington, DC: US Department of Health and Human Services; 2000.

36. Baier M, Calonge N, Cutter G, et al. Cancer Epidemiol Biomarkers Prev. 2000;9(2):229-232.

37. Hall HI, Van Den Eeden SK, Tolsma DD, et al. Testing for prostate and colorectal cancer: comparison of self-report and medical record audit. Prev Med. 2004;39(1):27-35.

38. Partin MR, Grill J, Noorbaloochi S, et al. Validation of self-reported colorectal cancer screening behavior from a mixed-mode survey of veterans. Cancer Epidemiol Biomarkers Prev. 2008;17(4):768-776.

39. Shields AE, Shin P, Leu MG, et al. Adoption of health information technology in community health centers: results of a national survey. Health Aff (Millwood). 2007;26(5):1373-1383. 